\title{
Philosophiques
}

\section{L'hypothèse du rêve et les vérités de la raison}

\section{Georges J. D. Moyal}

Volume 23, numéro 2, automne 1996

URI : https://id.erudit.org/iderudit/027407ar

DOI : https://doi.org/10.7202/027407ar

Aller au sommaire du numéro

Éditeur(s)

Société de philosophie du Québec

ISSN

0316-2923 (imprimé)

1492-1391 (numérique)

Découvrir la revue

Citer cet article

Moyal, G. J. D. (1996). L'hypothèse du rêve et les vérités de la raison.

Philosophiques, 23(2), 417-425. https://doi.org/10.7202/027407ar d'utilisation que vous pouvez consulter en ligne.

https://apropos.erudit.org/fr/usagers/politique-dutilisation/ 


\title{
l'HYPOTHĖSE dU RẼVE EI IES VÉrIIÉS DE LA RAISON
}

\author{
PAR \\ Georces J. D. Moral
}

Le texte qui suit n'est pas l'intégralité de celui que nous proposions en mai dernier lors du Congrès de l'ACFAS à Montréal. Pour diverses raisons - celles d'allocation d'espace ne sont que secondaires -, nous ne pouvons présenter ici qu'une des thèses que nous défendions à cette occasion. $I$ y en avait alors quatre :

I. L'hypothèse du rêve sape la perception des vérités de mathématiques et par conséquent celle des vérités de la raison.

II. Elle demeure opérante tout au long des Méditations.

III. Elle est adoptée parce qu'elle constitue un outil de doute bien plus efficace que celle de la folie pour mener $\dot{a}$ bien l'entreprise métaphysique. (Ceci s'oppose aux lectures - celle de H. G. Frankfurt ${ }^{1}$, par exemple - voulant que Descartes recule devant l'hypothèse de la folie parce qu'il ne veut ni ne peut se disqualifier lui-mēme, ni disqualifier ainsi son projet.)

$I V$. L'hypothèse du rève est la condition sine qua non de cette entreprise. Ceci parce qu'elle dote le méditant d'une liberté absolue d'assentiment, sans laquelle le projet métaphysique ne pourrait pas mème démarrer.

Parce que les trois autres la présupposent, nous ne nous concentrerons ici que sur la première. Nous ne la rapporterons à celles-là que selon les exigences d'une argumentation nécessairement condensée par endroits.

Le mieux est sans doute de commencer par un examen de la démarche par laquelle l'hypothèse du rêve se substitue à celle de la folie dans la $\Gamma^{r}$ Méditation ${ }^{2}$. Cette substitution, Descartes n'en donne pas les raisons, du moins pas explicitement. Frankfurt estime que ce qui motive l'abandon de la folie est que Descartes doit s'accorder un minimum de raison et exempter tout au moins les vérités fondamentales de la logique du doute - ce serait le cas notamment du principe de non-contradiction - , pour mener à bien son projet. S'il arrivait que certaines de ces vérités y soient néanmoins soumises, ce ne pourrait ètre que lorsqu'elles seraient perçues de façon obscure et confuse. Cette lecture est attrayante, certes, mais elle pose des difficultés sur lesquelles nous reviendrons.

Quels que soient les mérites de la thèse de Frankfurt, il convient de remarquer que le texte comporte deux ou trois indices assez révélateurs. Le premier en est l'allusion qui s'y fait à la nature corporelle : cerveau et vapeurs de la bile. Or, premièrement, on sait

1. Cf. : Démons, rêveurs et fous, Paris, 1989, trad. par Sylvain M. Luquet, de Demons, dreamers and madmen, Indianapolis et New York, 1970.

2. AT VII, 18-19; IX-1,14. 
que la folie est, dans une relation causale, l'effet d'un état corporel : celui de ces " noires vapeurs "sur le cerveau. Se supposer fou reviendrait donc à admettre un état de choses que Descartes, justement, tente de contourner:en effet, ses démarches dans la $I^{r}$ Méditation visent à rompre tout lien causal susceptible de déterminer sa pensée ${ }^{3}$. Deuxièmement, l'existence mème de la nature corporelle doit ètre révoquée en doute. Les doutes que susciterait l'hypothèse de la folie n'auraient plus alors aucun fondement, et dans ces conditions, un géomètre (athée ?) pourrait reprocher à Descartes d'avoir soumis les mathématiques et autres vérités de la raison à des doutes creux, dénués de fondement, qu'il n'aurait donc eu aucune raison d'envisager ni de craindre.

Le second indice révèle une raison plus importante de ce remplacement ; c'est ce que nous pourrions appeler le dogmatisme de la folie. En effet, le fou affirme (qu'il est roi, qu'il est fait de verre, etc.) ; et il ne s'embarrasse guère de doutes lorsqu'il le fait. Bien au contraire, il arrive souvent qu'il s'acharne à affirmer telle ou telle autre absurdité, qu'aucun raisonnement ne peut lui faire abandonner. Le doute mèthodique ou métaphysique, au contraire, exige du méditant qu'il se rende disponible à toutes les possibilités et que, pour ce faire, il ait pleine liberté de suspendre son jugement, de refuser son assentiment, etc. Le dogmatisme de la folie s'oppose donc diamétralement à l'ouverture d'esprit, au scepticisme foncier, que nécessite le projet métaphysique.

Le troisième indice tient au fait que l'hypothèse de la folie ait seulement été mentionnée. L'indice n'est pas négligeable : sachant qu'il allait la récuser, Descartes aurait fort bien pu s'abstenir d'en parler et passer directement à celle du rêve. Or Descartes s'est fixé pour projet de déterminer si la raison est fiable. Si par conséquent il a envisagé la possibilité qu'il soit fou, ce ne peut être que pour indiquer par là l'ampleur de la tâche qu'elle lègue à son successeur : le rève doit donc accomplir autant, sinon davantage, que l'hypothèse de la folie. Or la folie est absence de raison. Il faut donc que l'hypothèse du réve implique une révocation en doute de la raison et des vérités qui en émanent ou qui la constituent.

On voit dès lors ce que peuvent ètre les difficultés qui s'opposent à la lecture de Frankfurt :

1. Il faut, en effet, que l'hypothèse du rêve abatte au moins autant que ne le fait celle de la folie ; autrement, Descartes se serait rendu coupable d'avoir envisagé brièvement un doute touchant la raison et d'avoir reculé devant cette possibilité sans en donner explicitement la raison. Ce qu'une telle démarche aurait de subreptice, d'un côté, ce qu'elle lui ferait perdre en rigueur logique, de l'autre, parait tout à fait invraisemblable de sa part. Le rêve doit donc accomplir autant que la folie : il faut, par conséquent, qu'il sape les vérités de la raison.

2. Il n'est nulle part question, dans les textes, diune démarcation séparant les vérités de la raison exemptées du doute de celles qui ne le sont pas. Il est évident que certaines ne le sont pas : celles de

3. Descartes, selon nous, ne saurait admettre - tout au moins lors de sa quête métaphysique -, le psychologisme que certains, comme A. Kenny, lui attribuent (voir nos conclusions, en fin de texte). 
géométrie, par exemple, puisque Descartes les recouvre à la $V^{e}$ Méditation. Par ailleurs, le fait qu'il révoque en doute le principe de non-contradiction par l'intermédiaire d'un cas particulier ${ }^{4}$ ne prouve pas qu'il n'est alors perçu que de façon obscure et confuse. comme le voudrait Frankfurt : nous verrons bientôt quau contraire, Descartes le tient pour l'une des plus grandes certitudes dont il dispose alors.

3. Si effectivement Descartes s'était accordé un minimum de raison en tenant (au moins) certains principes de logique pour vrais, (a) il aurait alors eu des vérités dont il aurait pu extraire un critère sans s'embarrasser, comme il le fait dans les $I I^{e}$ et $I I^{e}$ Méditations, du doute métaphysique, du cogito, de la preuve de l'existence d'un Dieu vérace, etc. et (b) il lui aurait fallu admettre que la raison est fiable tout au moins dans la mesure où elle fournit de tels principes. Or (a) on ne le voit nulle part extraire un critère de la vérité de tels principes (en fait il dēnigre celui de non-contradiction et raille son inutilité5), et (b) il ne peut se permettre d'assumer la fiabilité de la raison : bien au contraire, c'est afin de révoquer en doute le critère mème des certitudes qu'elle offre dans ses opérations les plus simples qu'il fait intervenir un Dieu trompeur ${ }^{6}$.

4. Ou bien les vérités fondamentales de logique - dont Frankfurt prétend qu'elles sont exonérées du doute - s'avèrent éventuellement conformes au critère de clarté et distinction, ou bien elles ne le sont pas. On se serait attendu à ce que Descartes fasse état de leur conformité à ce critère lors de la remontée métaphysique s'il les avait exemptées du doute, ne fūt-ce que pour montrer qu'il avait bien eu raison de les en exempter. Or il ne fait rien de la sorte. Inversement, si elles n'étaient pas conformes à ce critère, cela voudrait dire que Descartes admet deux critères du vrai, ou tout au moins deux types distincts de vérités; or là encore, on ne lui voit rien dire de semblable.

En fait, plusieurs textes montrent que les vérités fondamentales de logique sont soumises au doute :

- Un passage de la IIT Méditation montre que le principe de noncontradiction est effectivement révoqué en doute par l'intermédiaire d'un cas particulier : "Me trompe qui pourra, [...] il ne saurait jamais faire [...] que quelque jour il soit vrai que je n'aie jamais été, étant vrai maintenant que je suis... " . Ce principe ne peut devoir sa vérité qu'à la contradiction qu'il y aurait à ce que "j'existe en ce moment $t$ " et "je n'existais pas au moment $t$ " soient simultanément vrais. Or c'est là un passage où Descartes fait ètat des plus grandes certitudes dont il dispose, au moment où il s'apprète à examiner - et éventuellement fonder - la fiabilité de ce qui les identifie comme telles : le critère de clarté et distinction. Si par conséquent ce critère n'a pas encore été

4. Il s'agit de l'impossibilité où serait Dieu de faire en sorte que "...quelque jour il soit vrai que je n'aie jamais été, étant vrai maintenant que je suis..." III $I^{e}$ Méditation, AT VII, 36 ; IX-1, 28.

5. Dans sa lettre à Clerselier de juin ou juillet 1646 (AT IV, 444-445).

6. Nous verrons plus loin pourquoi il convient de distinguer le contenu de nos certitudes, que disloque le rève, du critère nous permettant de les identifier et dont la révocation en doute exige l'intervention du Dieu trompeur.

7. Cf. n. 4, supra. 
validê, elles ne peuvent être tenues pour des vérités à ce moment-là et sont alors douteuses ${ }^{8}$.

- La lettre à Mersenne du 15 avril 1630 : "On vous dira que si Dieu avait établi ces vérités, il les pourrait changer comme un roi fait ses lois ; à quoi il faut répondre que oui, si sa volonté peut changer [...] et généralement nous pouvons bien assurer que Dieu peut faire tout ce que nous pouvons comprendre, mais non pas qu'il ne peut faire ce que nous ne pouvons pas comprendre...9". Or du moment que l'êternité des vérités èternelles dépend de l'immutabilité de la Volonté qui les a créées, et que l'existence même de Dieu est révoquée en doute (ou qu'il se substitue à lui un Trompeur tout aussi puissant), il y a lieu de se demander ce que valent alors ces vérités, ce qu'il advient de leur éternité. D'ailleurs, si ce que Dieu peut faire dépasse notre compréhension et si l'une des conditions de cette compréhension est le principe de non-contradiction mème, il devient d'autant plus vraisemblable que ce principe soit nécessairement révoqué en doute.

- Une lettre à Clerselier ${ }^{10}$ fait bien voir que Descartes, reconnaissant deux types de principes : les notions communes et les principes existentiels de sa métaphysique, estime les premiers, parmi lesquels il nomme celui de non-contradiction, comme étant superflus et de nul usage dans la recherche de ce qui existe. Ceci s'oppose de front à ce qu'affirme Frankfurt selon qui Descartes n'aurait pu s'en départir parce qu'il en aurait eu besoin pour guider ses démarches.

On pourrait objecter ici que ce n'est pas l'hypothèse du rève seule qui suffit à abattre les vérités de mathématiques, mais qu'il convient de lui adjoindre celle du Dieu trompeur ou celle du malin génie. Ce qui est exact : en effet, le rève disloque les vérités de la raison mais nous ne nous en apercevons pas et ne pensons pas en ètre moins certains pour autant. Or il nous est impossible de distinguer l'éveil du rêve ; il convient alors de révoquer en doute non pas les contenus divers de nos certitudes, mais leur critère mème - la clartè et distinction des perceptions que nous en avons - par le seul moyen qui nous permette de contourner cette incapacité : l'invocation d'un Trompeur seul susceptible de connaître notre erreur. On voit cependant que mème s'il faut lui adjoindre celle d'un Trompeur, l'hypothèse du rève accomplit bien plus qu'on ne l'a admis jusqu'ici.

Notre thèse se défend-elle? Est-il possible que le réve disloque et déforme les vérités de mathématiques? Elle s'oppose, bien entendu, à l'opinion reçue, ainsi qu'au texte bien connu sur lequel celle-ci se fonde : "Car, soit que je veille ou que je dorme, deux et trois joints ensemble formeront toujours le nombre de cinq, et le carré n'aura jamais plus de quatre cótés..." "

Ce texte semble d'une parfaite univocitè. Il y a néanmoins des raisons de penser qu'il est ambigu en fait. Elles sont de quatre ordres

8. Il n'y a d'ailleurs que ce passage qui puisse correspondre à la référence, dans l'Entretien avec Burman. AT V, 146, aux "principes universels ou axiomes, par exemple qu'il est impossible qu'une mème chose soit et ne soit pas " et dont Descartes nie qu'il ait omis d'en douter.

9. AT I, 145-146.

10. Celle de juin ou juillet $1646: c f$. n. 5 , supra

11. AT VII, 20 ; LX-1, 17. 
différents : (a) d'ordre indépendant (c'est-à-dire qu'elles relèvent du genre de choses que l'on peut attribuer à quiconque et par conséquent à Descartes aussi), (b) d'ordre contextuel, (c) d'ordre textuel, et finalement, (d) d'ordre systémique.

\section{(a) Les raisons d'ordre indépendant}

Le rêve, c'est bien connu, opère des réassemblages (chevaux ailés, chimères etc.) et disloque. Or il nous semble que ces dislocations contreviennent non seulement aux lois de la nature, mais aussi aux contraintes conceptuelles et logiques qui régissent notre entendement en temps ordinaire. La meilleure preuve de ceci nous en est donnée par certaines gravures de M. Escher - Montée et descente en est un exemple frappant - lesquelles représentent des impossibilités logiques. Or si de telles représentations peuvent occuper notre champ visuel durant l'éveil, n'est-il pas a fortiori possible et plausible qu'elles le fassent en rêve ? Du reste, pourquoi n'en seraitil pas ainsi ? Il semble tout à fait plausible de rêver que, ayant demandé, au guichet d'une banque, la monnaie d'un billet de $5 \$$, l'on reçoive en échange un billet de 2 \$ qu'accompagne un billet de $37,26 \$$ ou mème un superbe poisson (que l'on ne pense pas avoir acheté ainsi ${ }^{12}$ ), et que l'on trouve la chose parfaitement normale. Nous verrons d'ailleurs que Descartes eut connaissance d'un cas semblable et que, loin de le récuser, il l'admit comme tel.

\section{(b) Les raisons d'ordre contextuel}

Descartes vient de supposer qu'il est endormi et qu'il rève après qu'il s'est aperçu qu'il n'a aucun moyen de distinguer l'éveil du rêve, ni de savoir dans quel état il se trouve à présent. Or rien n'est intervenu entre-temps qui suggère que cette supposition ait été abrogée. On peut alors resituer ce passage dans son contexte à peu près dans les termes suivants: "Je viens de supposer que je suis endormi et que je réve. Or ce n'est là qu'une supposition et je ne puis savoir ce qu'il en est en fait. Quoi qu'il en soit, c'est-à-dire, " soit que je veille ou que je dorme, <il me semble en ce moment que> deux et trois joints ensemble formeront toujours le nombre de cinq, et le carré n'aura jamais plus de quatre côtés"'13". La lecture que nous en faisons ainsi montre bien que, contrairement à ce qui en a èté admis généralement, ce texte soumet à caution les perceptions qu'a le méditant des véritès de mathématiques au moment où il les rapporte. Il les soumet donc à un doute, celui que suscite l'incertitude concernant son état d'esprit : éveil ou rêve.

Sans doute nous sera-t-il reproché d'avoir inséré les quelques mots entre crochets obliques : rien, semble-t-il, n'autorise une telle démarche. Il nous semble au contraire que deux choses la justifient

12. Il n'y aurait là qu'une autre forme de la dislocation onirique : en l'occurrence, celle-ci serait conceptuelle, puisque le rève conférerait ainsi cours légal à cet animal.

13. Cette formule trouve d'ailleurs son écho dans le cas des perceptions sensorielles : "Enfin je suis le mēme qui sens, c'est-à-dire qui reçois et connais les choses par les organes des sens, puisqu'en effet je vois la lumière, j'ouïs le bruit, je ressens la chaleur. Mais I'on me dira que ces apparences sont fausses et que je dors. Qu'il soit ainsi, à tout le moins, il est très certain qu'il me semble que je vois, que j'ouis et que je m'échauffe..." . AT VII, 29 ; IX-1, 23 ; nous soulignons. 
amplement : (a) Descartes ne prétend pas rapporter ici ce qui en est des mathématiques, mais bien plutôt ce qu'il en est des perceptions qu'il en a ; cela est d'autant plus vraisemblable que (b) il informe ses seconds objecteurs qu'il ne s'est servi que de la méthode analytique, laquelle consiste à remonter des effets - en l'occurrence, ses perceptions : les seules choses dont il dispose alors - à leurs causes $^{14}$.

\section{(c) Les raisons d'ordre textuel}

La thèse, communement admise, de l'immunité des mathématiques au doute qu'occasionne le rêve n'est nulle part si nettement désavouée que dans les Septièmes objections et réponses. C'est d'ailleurs là que fait son apparition le cas, mentionné il y a un instant, d'une dislocation onirique accompagnée de certitude, que Descartes aurait reconnue et admise pour telle. En effet, dans ses objections, le P. Bourdin montre qu'il a cru comprendre, lui aussi, que le rêve n'entame pas les vérités de mathématiques. Il écrit ceci :

Mais, dites-vous, il en est bien autrement des choses qui sont tout à fait certaines ; car elles sont telles, qu'à ceux mème qui dorment ou qui sont fous, elles ne peuvent jamais paraitre douteuses. - Est-ce donc tout de bon, je vous prie, que vous dites que les choses tout à fait certaines sont telles qu'elles ne peuvent pas même paraitre douteuses à ceux qui dorment ou qui sont fous ? Mais, enfin, où les trouverez-vous, ces choses ? Et pourquoi, s'il est vrai qu'à ceux qui dorment ou qui ont l'esprit troublé, les choses qui sont ridicules et absurdes leur paraissent cependant quelquefois non seulement vraies, mais aussi très certaines, pourquoi aussi celles qui sont les plus assurées ne leur paraitront-elles pas fausses et douteuses? Et, pour preuve de ceci, j'ai connu une personne qui, un jour, comme elle sommeillait, ayant entendu sonner quatre heures, se mit à compter ainsi l'horloge, une, une, une, une. Et pour lors l'absurdité qu'elle concevait dans son esprit la fit s'écrier : "Je pense que cette horloge est dérangée, elle a sonné quatre fois une heure ${ }^{15}$."

Ce à quoi Descartes, après en avoir retranscrit la première phrase, répond, infligeant ainsi à cette thèse un démenti on ne peut plus formel :

Je ne sais par quelle analyse cet homme subtil a pu déduire cela de mes écrits ; car je ne me ressouviens point d'avoir jamais rien dit de tel, ni mème rêvé en dormant. Il est bien vrai qu'il eût pu conclure de mes écrits que tout ce qui est clairement et distinctement conçu par quelqu'un est vrai, encore que celui-là cependant puisse douter s'il dort ou s'il veille, ou

14. D’ailleurs lui faire dire qu'il parle des vérités de mathématiques ellesmēmes, plutöt que des perceptions qu'il en $a$, mène à des difficultés de toutes sortes dont celle-ci, par exemple : comment pourrait-il, au moment oú il vient d'admettre qu'il ne peut distinguer l'éveil du rêve, prétendre avoir comparé ses certitudes rèvées avec celles de l'éveil pour s'estimer à mème d'affirmer qu'elles ne varient pas, qu'elles sont identiques et que dans un cas comme dans l'autre, $2+3=5$ ? En somme, Descartes, ne fait que rapporter là une certitude subjective (l'expression est de Mme G. RodisLewis), dont le contenu doit, dès lors, ètre soupçonné d'avoir subi une distorsion.

15. AT VII, 457. La traduction par Clerselier des Objectiones septimæ ne paraissant pas dans AT, nous renvoyons à l'édition en trois tomes, par F. Alquié, des CEures philosophiques de Descartes, Paris, 1963-1973, Alquié II, 954 . 
mème aussi, si l'on veut encore qu'il dorme ou qu'il ne soit pas en son bon sens; parce que rien ne peut être clairement et distinctement conçu par qui que ce soit qu'il soit tel qu'il le conçoit, c'est-à-dire qu'il ne soit vraí. Mais, parce qu'il n'appartient qu'aux personnes sages de distinguer entre ce qui est clairement conçu et ce qui semble et parait seulement l'être. je ne m'étonne pas que ce bon homme prenne ici l'un pour l'autre ${ }^{16}$.

En opérant, dans cette dernière phrase, la distinction entre ce qui est clairement et distinctement perçu et ce qui semble seulement l'être. Descartes fait bien voir qu'il n'exclut nullement la possibilité d'éprouver, en rêve comme en éveil. des certitudes ayant pour contenu des faussetés. De plus, le fait qu'il reconnaisse ici que les dislocations du rêve (ou de la folie) soient en effet susceptibles de lier clarté et distinction à des absurdités dans son esprit prouve que son intention, dans la $I^{e}$ Méditation, était bien de soumettre à caution sa constatation de certitude au sujet des mathématiques. Comme par ailleurs, il n'opère nulle part de distinction ${ }^{17}$ entre les vérités de mathématiques et les autres vérités de la raison, il devient d'autant plus invraisemblable que ces dernières aient été exemptées de ce doute.

\section{(d) Les raisons d'ordre systémique}

La réponse de Descartes à Bourdin met en lumière la conception que se fait Descartes de sa propre métaphysique : il s'agit d'une philosophie critique, c'est-à-dire d'une recherche des conditions de (la possibilité de) la connaissance. Il s'agit donc pour lui de fournir le tracé de la forme de la connaissance. Dès lors les termes-clefs que comporte ce tracé ne peuvent ètre définis matériellement euxmèmes; ils ne peuvent qu'ètre situés les uns par rapport aux autres, dans ce qu'on pourrait appeler une cartographie de la connaissance : tant que cette recherche n'a pas encore abouti, ils ne peuvent opérer que comme marque-places ${ }^{18}$. Tout au plus avons-nous droit à une définition ostensive des vérités claires et distinctes : celles de géométrie, dans la $V^{e}$ Méditation.

La réponse de Descartes au P. Bourdin confirme le caractère critique des Méditations. Elle comporte deux choses :

(a) d'abord l'affirmation, sur le plan formel, que le clair et distinct est vrai où qu'il apparaisse : en éveil comme en rêve :

(b) ensuite la reconnaissance que sur le plan matériel, certaines choses sont fausses que néanmoins nous pensons avoir perçues clairement et distinctement (là encore, en rève comme en éveil) ; Descartes avertit qu'il n'est pas donné à tout le monde de repérer lé

16. AT LX-1, 461-462. Alquié II, 960-961. Nous soulignons.

17. Bien au contraire, il aurait plutôt tendance à assimiler les lois de la nature aux vérités de la raison. Voir sa lettre à Morus de février 1649, AT V, 275.

18. Nous en avons la preuve dans le fait quaucun des termes épistémiques n'est en fait défini dans les Méditations: vérité, évidence, certitude, doute, indubitabilité, connaissance, et surtout clarté et distinction. Il n'y a rien de particulièrement nouveau à cela : Aristote en fait autant dans les premiers livres de l'Éthique à Nicomaque, où les termes de bonheur, activité, vertu. âme, etc. sont bien reliés les uns aux autres, mais n'y sont pas définis : les moyens d'en reconnaitre l'extension ne nous y sont pas donnés, et il faut attendre la fin du livre $X$ pour apprendre enfin en quoi consiste lactivité de l'âme que désigne le mot de bonheur. 
clair et distinct hic et nunc, c'est-à-dire, dans la pratique, dans la mise en œuvre du critère, et que cela exige une certaine sagesse.

Or l'essentiel de la réponse à Bourdin est que Descartes y nie s'être engagé sur le plan matériel, ou si l'on préfère, sur le plan pratique. Ce qui fait que dans la mesure où, lorsque Descartes écrit : "...soit que je veille ou que je dorme, deux et trois joints ensemble formeront toujours le nombre de cinq...", il ne peut qu'y rapporter une certitude du moment, il avertit qu'il est possible que son sommeil (supposé) en ait disloqué le contenu.

Il est remarquable que la réponse à Bourdin ait été complètement négligée des commentateurs. Cependant deux choses semblent avoir contribué à cela et avoir perpétué par là le maintien de l'interprétation communément admise, c'est-à-dire la thèse de l'immunité des mathématiques au rêve :

(a) En un sens, le fait que ce texte donne pour exemples des vérités (que $2+3=5$ et que le carré a 4 cótés). Ce qui est tout à fait accidentel: il se trouve que ce sont tout simplement de celles qui se présentaient à Descartes à ce moment-là. Rien n'excluait en principe qu'il y ait énoncé des absurdités à leur place ; rien, si ce n'est, bien entendu, qu'il s'agissait justement de relativiser ses perceptions les plus claires et les plus distinctes pour les dévaloriser, les ravaler au niveau de celles du réve et les rendre ainsi d'autant plus suceptibles d'être reconnues douteuses par ses lecteurs. Ce n'est qu'en cet autre sens qu'il n'y aura rien eu d'accidentel à son choix d'exemples.

(b) Une confusion : on continue de lire les Méditations comme s'il s'agissait d'un guide pratique pour l'obtention de certitudes alors qu'il s'agit en fait d'un ouvrage de philosophie critique. La référence aux "personnes sages "dans la réponse à Bourdin, ainsi que l'absence de toute définition du clair et distinct montrent bien que Descartes ne cherche nullement à fournir un guide pratique, puisqu'il n'explique nulle part comment s'acquiert cette sagesse, ni à quoi se reconnait le (réellement) clair et distinct.

Nos conclusions ne pourront ètre ici que partielles :

Dans le texte qui nous occupe ici - celui qui a servi de socle à la thèse de l'immunité des mathêmatiques au doute --, Descartes ne fait que constater qu'il éprouve des certitudes en rêve comme en éveil : mais il ne va pas jusqu'à affirmer que le rève ne saurait en disloquer le contenu. S'il en est bien ainsi, c'est donc que l'hypothèse du réve est au moins aussi puissante que celle de la folie dans son ouvre dévastatrice : le rêve peut déformer tout, y compris les vérités de la raison, et il porte en lui, tout comme la folie, des convictions, des sentiments de certitude. La dislocation n'étant qu'un réassemblage, ce réassemblage peut lier des sentiments de certitude à la perception du contingent, du faux, ou mème de l'incohérent ; inversement, il peut nous faire prendre des vérités de mathématiques pour des contingences (ce qui est là, en fait, l'essentiel de l'objection de Bourdin). Disons aussi que ces sentiments de certitude se traduisent tout simplement par l'incapacité où l'on se trouve de se représenter la négation de ce qui parait clair et distinct. Cette incapacité peut n'être que psychologique, ou momentanée - auquel cas nous n'avons affaire qu'à un semblant de clarté et distinction - ; mais elle peut aussi 
nous être imposée par une incontournable volonté divine - auquel cas nous avons affaire, bien entendu, à une vérité.

Cependant l'hypothèse du rêve ne sape pas tout: elle ne sape pas la fiabilité du critère de clarté et distinction : tout au moins ne le fait-elle que dans la mesure où Descartes ne peut distinguer l'éveil du rêve. S'il doit établir la fiabilité de la raison, Descartes ne peut s'en tenir à l'hypothèse du rêve : car un géomètre qui estimerait le doute metaphysique extravagant et ridicule, pourrait refuser de l'y suivre, et lui recommander de s'assurer, autant que faire se peut en pratique, qu'il ne dort pas et de se fier plutôt à ce que lui livreraient alors ses perceptions claires et distinctes, sans s'embarrasser d'un doute affectant la plus haute évidence que puisse embrasser l'entendement humain. Il lui faut donc révoquer en doute le critère de clarté et distinction dans ses conditions d'emploi optimales : c'est à cette fin que Descartes invoque le Dieu trompeur, auquel il substitue presque aussitôt le malin génie.

A cet égard, la fonction de l'hypothèse du rêve est de suggérer que tout comme les certitudes éprouvées en rêve peuvent avoir un contenu déformé, de méme les perceptions claires et distinctes de l'éveil peuvent, elles aussi, être la dislocation de vérités nous demeurant inconnues et que seuls connaissent Dieu et les anges : la vie peut en somme n'ètre qu'un songe. (Ce qui suppose, bien entendu, la théorie des idées représentatives.)

Ceci implique donc un doute embrassant la fiabilité des représentations que Dieu détermine en nous, étant donné que nous ne pouvons concevoir certaines choses autrement que nous ne le faisons : il s'agit donc de mettre ici à l'épreuve les lois mémes de l'entendement. Par là est révoqué en doute le psychologisme dont Kenny ${ }^{19}$ fait la clef de voûte de la métaphysique de Descartes.

S'il en est effectivement ainsi, il est évident qu'en servant à révoquer en doute la raison mème et ses principes constitutifs, l'hypothèse du rêve accomplit autant que ne l'aurait fait l'hypothèse de la folie : les déformations qu'elles suscitent toutes deux se ressemblent. Il se trouve cependant qu'elle accomplit bien plus, puisqu'elle ouvre la voie ${ }^{20}$ à une liberté infinie indispensable à la mise en œuvre du doute métaphysique : en ceci elle surpasse de loin sa rivale, puisqu'elle autorise ainsi une gestion réfléchie et délibérée du projet métaphysique ; ce à quoi s'oppose, justement, le déterminisme auquel est soumise la folie.

Département de philosophie

Collège universitaire Glendon, Université York.

19. Dans " Descartes on the will ", Kenny écrit ceci : " To abandon the theory that clear and distinct perception necessitates the will is to call in question the whole validation of reason in which the Meditations culminates. "dans R. J. Butler, ed., Cartesian studies, Oxford, 1972, p. 29.

20. Ceci se fait au tout dernier paragraphe de la $I^{\text {re }}$ Méditation, AT VII, 23 : IX$1,18$. 